\title{
AN EXTRACTION APPROACH OF THE TOP-BOUNDED SPACE FORMED BY BUILDINGS FOR PEDESTRIAN NAVIGATION
}

\author{
J. Yan ${ }^{1}$, A. A. Diakite ${ }^{1, *}$, S. Zlatanova ${ }^{1}$ \\ ${ }^{1}$ UNSW Built Environment, Red Centre Building, Kensington NSW 2052, Sydney, Australia \\ jinjin.yan@student.unsw.edu.au, (a.diakite, s.zlatanova)@unsw.edu.au
}

Commission IV, WG IV/5

KEY WORDS: Top-bounded, Space, Generating, Buildings, Pedestrian, Navigation

\begin{abstract}
:
The navigation of pedestrians can be regarded as their movements from one unoccupied space to another unoccupied and connected space. These movements generally occur in three types of environments: indoor, outdoor, and semi-bounded (top-bounded, and/or side-bounded) spaces. While the two former types of spaces are subject to most of the attention, the latter (semi-bounded) also presents a valuable impact on the navigation behaviour. For example, top-bounded environments (e.g. roofs, shelters, etc.) are very popular for pedestrian navigation since a top structure can offer protection from harsh weather, rain, or strong sun. However, such semibounded spaces are completely missing in current navigation models and systems. This is partly explained by the fact that modelling the space, which is by defining a three-dimensional boundless and extensible component (mainly out of the indoor environment), is a very challenging task. In this paper, we propose a structure-based approach for top-bounded space extraction in the built environment, relying on 3D models. Thanks to the rapid expansion and availability of $3 \mathrm{D}$ city models, our approach can help to account for such type of spaces in 3D pedestrian navigation systems.
\end{abstract}

\section{INTRODUCTION}

Navigation, also called path-finding or way-finding, is a fundamental activity for human beings. It is described as the method of determining the direction of a familiar goal across unfamiliar terrain (May et al., 2003), or the process of orientation to reach a specific distant destination from the origin (Krūminaite and Zlatanova, 2014). With the high and increasing complexity of the urban environment, more and more navigation systems are developed to assist people in this essential task. For those tools to be able to perform correct pedestrian navigation, a model representing the structuring of the environment (space) and describing semantic information of the elements it contains have to be available. While 2D models have been traditionally regarded as enough to support the navigation of pedestrian, recent researches have emphasized on the wide enhancement that relying on a 3D model could bring to the task (Diakité and Zlatanova, 2018). From this, a 3D space model becomes a critical asset for pedestrian navigation. In addition to the space model, several other components are needed, such as 3D localization of start point and destination, a 3D model that represents the space subdivision, 3D algorithms for path computation (on a topological model or a grid), guidance (Points of Interest, Landmarks), visualization of the path, and finally tracking/correction (if the path is not followed) (Zlatanova et al., 2014, Worboys, 2011).

There are three types of environments involved in pedestrian navigation: indoor (bounded), outdoor (unbounded), and semi-bounded spaces. While all three environments are strongly interrelated, there are not always clear boundaries between them and pedestrians can move seamlessly into them (Nagel et al., 2010). In the built environment, they are generally resulting from built structures. Pedestrians have well-defined preferences on how to pass through such environments and those preferences may depend on

${ }^{*}$ Corresponding author multiple factors, e.g. time of the day, weather conditions, emergency situations or even gender differences (Melson, 1977). In this respect, environments which are not strictly defined as indoor or outdoor can play an important role in navigation options for pedestrians. They may prefer to use top-bounded environment for some additional purposes, e.g. growing plants (Kim et al., 2011), learning activities (Nasir et al., 2014), adapting to thermal environments (Lin, 2009), sheltering from the sun and wind (He and Hoyano, 2010, Spagnolo and Dear, 2003), and improving the physical environment of the markets (Kim et al., 2008). For instance, people may prefer to navigate through indoors for as long as possible in harsh weather, while during nice weather they would prefer walking outdoors. In rainy periods, they may prefer to choose paths with overhanging roofs/shelters, as these structures can protect them from the rain (Koide and Kato, 2005). Often such environment act as connections between indoor and outdoor environments, an approach which has been used to improve residential comfort and reduce cooling and heating energy requirements (Kim et al., 2011), or a special structure to obtain a good building micro-climate (Du et al., 2014). Moreover, the micro-climate of partially enclosed environments often has a better thermal sensation (usually lower effect of wind, less heat) than the outdoor (Pagliarini and Rainieri, 2011b). Therefore, the topbounded environment is critical in the user-adaptive pedestrian navigation.

However, despite the valuable contribution of semi-bounded spaces in the pedestrian navigation task, they are non-existent in currently available navigation systems. This is mainly because the latter often rely on a very simplified model which consider only the ground as a relevant structure for the purpose. On the other hand, the difficulties involved in the definition of those sub-spaces with no clear boundaries may have also discouraged any further implication of such parameters in the proposed navigation solutions. Nevertheless, thanks to the growing availability of 
3D model standards such as CityGML (Gröger et al., 2008) and IFC (Building SMART International, 2013), it becomes possible for navigation tools to account for such types of semi-bounded spaces. In this paper, we focus on top-bounded spaces. We identify the relevant structures of the urban environment from which the top-bounded spaces are resulting, and we propose a structurebased approach to automatically generate them in spatial models. The approach assumes a geometric and semantic description of the relevant structures.

After exploring the related work in the next section, we discuss the different categories of spaces inferred by buildings, emphasizing on the top bounded ones, in section 3 . Section 4 is dedicated to the details of our extraction approach and section 5 brings the related implementation and experiments. We conclude in section 6 with an overall discussion and suggestions for future work.

\section{RELATED WORK}

When it comes to the study of semi-bounded spaces in the built environment, the large majority of the available literature has been dedicated to the domains of thermal comforts (He and Hoyano, 2010, Spagnolo and Dear, 2003, Kim et al., 2011, Du et al., 2014, Pagliarini and Rainieri, 2011b). Those researches generally focused on the assessment of architectural design for residential comfort improvement and spatial design for good building micro-climate, supporting thereby further reduction of energy demand for thermal comfort. It is also noticeable that the semibounded spaces are being given different names, and the reader is often left without a clear definition of their meanings and differences. For instance, the notions of semi-indoor, semi-outdoor, semi-enclosed, semi-opened, transitional spaces, and transition zones are the most commonly used. From a structural point of view, the roof (or shelter) is the upper boundary of an environment. Therefore, in this paper, spaces below them are referred to as top-bounded spaces. More precisions to our definitions will be provided in the next section.

Several works have been oriented towards top-bounded spaces created by adding the roof (shelter) to the outdoor space (Turrin et al., 2010). This is because such structure plays a key role in contributing to the climate control. For example, (Turrin and Timmeren, 2009) investigated the design of the Vela Roof, which was meant to cover an outdoor space. The author pointed out the main weaknesses of the structure and proposed few improvements, including active solar technologies and passive systems for heating and cooling. (Taj and Blocken, 2009) proposed an analysis of the ventilation effect induced by the removable roof of the Amsterdam Arena stadium, which by that has the property to become closed or semi-opened stadium.

In South Korea, enclosed arcades have been applied to traditional markets in order to improve the physical environment of the markets (Kim et al., 2008). A specific group of semi-outdoor spaces can be identified as covered by large roofs or partially open envelopes, leaving a relevant direct connection with the outdoor environment (Turrin et al., 2012). Semi-outdoors is defined in (Ruey-LungHwang and Tzu-PingLin, 2007) as "exterior spaces that are sheltered and attached to the building". Glass roofing is used to produce a semi-outdoor urban space by sheltering it from the environment without excessively attenuating the natural lighting (Pagliarini and Rainieri, 2011a). (Lin et al., 2006) studied bus shelters, which they defined as semi-outdoor spaces with shelter provided in the form of a roof. The authors stated that the thermal comfort of bus shelters is as important as that of buses themselves in improving the quality of the experience of bus travel. (Kray et al., 2013) defined the spaces, generally located between indoor and outdoor, and can be neither consistently classified as being indoors nor being outdoors and that share property with either category, as transitional spaces. Another similar definition is transition zones in (Winter, 2012). The top-bounded spaces are included in these definitions, but it is still cannot clearly distinguish if the top of the spaces is enclosed or sides are enclosed.

To the best of our knowledge, there is no study dealing with the pedestrian navigation aspect of the semi-bounded spaces. As previously said and considering the thermal comfort parameters involved in the task, there is definitely a clear added-value in identifying such spaces for a pedestrian navigation system. After providing details on the definitions of the spaces involved in the process, we provide an approach to extract the top-bounded spaces in existing 3D spatial models.

\section{SPACES FORMED BY BUILDINGS}

The built environment subdivides the space into three categories: indoor, outdoor and semi-bounded spaces. Among them, the indoor and the semi-bounded ones are generally originating from buildings, but not only (e.g. they can also result from vegetations, rocks, etc.). We also distinguish among the semi-bounded spaces the top-bounded and the side-bounded ones. We give the definition of all those spaces below.

Spaces formed by built structures are the hollow (unoccupied) parts of indoor, top-bounded, and side-bounded environments, and people can have activities in them. Although the space may also be defined as a boundless structure, it is necessary to provide its boundaries in a spatial model so that local specificities can be accounted. We, therefore, define the discussed environments based on their identified boundaries.

The indoor environment is physically enclosed by the building components, such as roof, floor, and walls (doors, windows). That is, indoor space surely has top(s) and sides, and it is enclosed completely by a top(s) and sides, see Figure 1(a).

The top-bounded environment is semi-opened to the outdoor, but physically enclosed on the top by roof or shelter. Specifically, the top-bounded space surely has top (or roof), but not physically enclosed completely by the roof and walls. For instance, the bus stand is a top-bounded space with sides (Figure 1(b)), but the gas station is also a top-bounded space without sides (Figure 1(c)). The top of the top-bounded environment can come from different structures, e.g., indoor environment (Figure 1(e)), balconies (Figure 1(f)).

The Side-bounded environment is also semi-opened to the outdoor, but it is enclosed by sides rather than the top. Example of the side-bounded space can be seen in Figure 1(d), which surely has no top(s), but has sides.

In summary, the characteristics of the environments (spaces) formed by built structures are related to the top(s), side(s), and whether they are enclosed completely (see Table 1).

For a spatial model to be able to account for all those different spaces, it is necessary to represent them as objects of the 


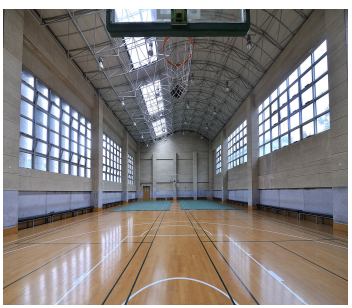

(a) Indoor

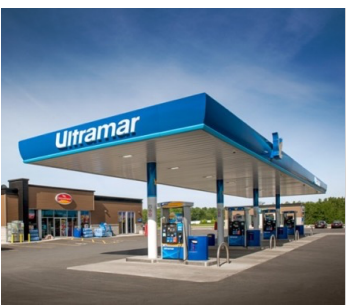

(c) Top-bounded

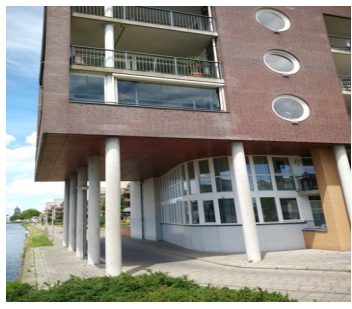

(e) Top-bounded

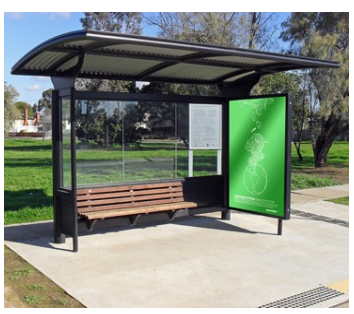

(b) Top-bounded

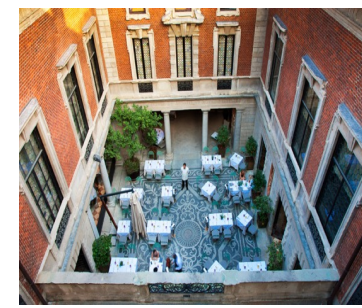

(d) Side-bounded

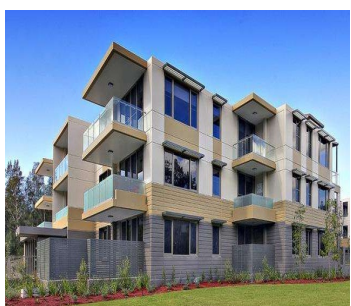

(f) Top-bounded
Figure 1. Examples of different environments (spaces) formed by buildings.

model. Most of the existing models on which pedestrian navigation can rely possess their own definition of spaces. For example, in the IFC standard, a space (which is represented by the If Space class) represents an area or volume bounded actually or theoretically. It provides for certain functions within a building (it is then linked to a storey) but it can be exterior to it as well (linked to the construction site) (Building SMART International, 2013). Thus, a space entity needs to be geometrically bounded to be represented in the model. Similarly, although there is nothing such as a space object in CityGML, an equivalent class would be a Room, which is regarded as a semantic object for modelling the free space inside a building and should be uniquely related to exactly one building or building part object (Gröger et al., 2008). Therefore, an extension of that notion is necessary to bring such space objects outside of the indoor environment. A closely related standard to CityGML, namely Indoor Geography Markup Language (IndoorGML) (Lee et al., 2014) provides more adapted spatial models for navigation purposes, and its definition could be adapted for environment other than the indoor as well.

\begin{tabular}{|l|c|c|c|}
\hline Types & Top(s) & Side(s) & Enclosed Completely \\
\hline Indoor & Yes & Yes & Yes \\
Top-bounded & Yes & Yes/No & No \\
Side-bounded & No & Yes & No \\
\hline
\end{tabular}

Table 1. Characteristics of environments (spaces) formed by built structures.

The previously cited standards can still provide a description of the structural building elements necessary to generate the space objects. Here, we limit our focus to the top-bounded spaces resulting from buildings for the purpose of pedestrian navigation. We will investigate top-bounded spaces induced by buildings indoor and outdoor.

\section{EXTRACTION APPROACH OF THE TOP-BOUNDED SPACE}

Starting from a 3D model describing a built environment, our top-bounded space extraction approach includes the following five steps: (a) Identification of the relevant building parts; (b) Elevation-based sorting of the components (surfaces); (c) Detection of upper/lower space boundaries; (d) Space generation; (e) Space trimming. Figure 2 illustrates the different phases of the workflow.

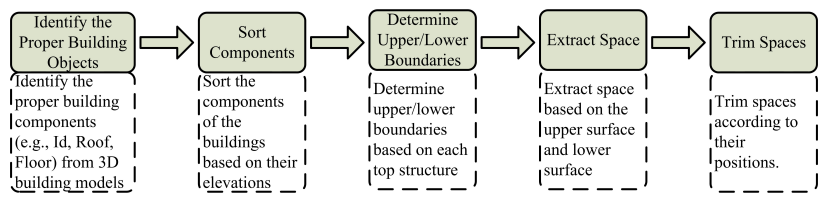

Figure 2. The workflow of the space extraction.

\subsection{Identification of Proper Building Objects}

This step is related to the data sources (inputs). The building generally consists of several components (Neufert et al., 2012): foundations, walls, floor slabs, roofs, windows, glasses, doors, stairs, escalators (lifts or elevators), moving walkways, etc. Among those components, some are particularly relevant to our case.

In general, building elements like balconies, dormers or outer stairs, not only strongly affect the outer appearance of a building, but also can result in top-bounded spaces for pedestrians. Therefore, the first step is to detect those building components in the input 3D model, such as roofs, floor slab, and floors. The ground also necessary, especially in the model who has no floors. For instance, the gas station has roof only. For this purpose, we strongly rely on the semantic description provided in the model, thus we assume an advanced level of detail (e.g. minimum CityGML LoD3 or IFC).

From a geometric point of view, the building objects (surfaces) are not limited to quadrilaterals, i.e., they could be polygons, but all vertices of a single polygon are coplanar. Furthermore, all of the surfaces, including the ground surface, can be tilted at any angle, and the surfaces from buildings can float over or sink into the ground surface (terrain).

\subsection{Components Sorting Based on Elevation}

After selecting the relevant building components, we sort them on the basis of their elevation (height values) in the vertical direction. We rely on the Cartesian $Z$-axis as the direction of reference, the ground level is considered as the origin.

The elevation of each component is computed using equation 1:

$$
H_{i}=\frac{1}{n} \sum_{i=1}^{n} z_{i}
$$


where

$$
\begin{aligned}
& H_{i}=\text { elevation of component } \\
& n=\text { the number of components vertices } \\
& z_{i}=\text { the } z \text { of vertices }
\end{aligned}
$$

This approach allows to also consider inclined component like roofs, whose orientation may present an non-zero angle against the ground. In the case where several components have the same elevations, they can still be processed separately in the next steps.

\subsection{Determination of Upper/Lower Space Boundaries}

This step is determining the upper/lower boundaries of the targeted top bounded spaces. We assume that we have a list of all the relevant components sorted from the previous step. Based on a projection approach, from higher to lower components (in the direction of $Z$-axis), we detect overlaps that symbolize the sought space boundaries. At this stage, we only rely on surface objects, thus if the building components are volumes (e.g., in IFC), a preprocessing step to this one will include a decomposition of the volumes into relevant upper and lower surfaces as well.

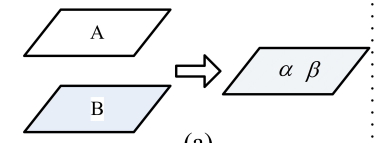

(a)

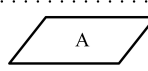

$\triangle \mathrm{B}$

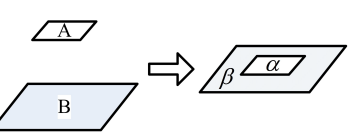

(b)

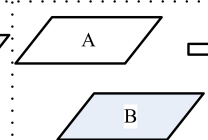

(c)

(d)

Figure 3. Four different cases of projections with overlaps.

Specifically, all of components (including the ground surface) are projected onto a virtual geometric XY plane along Z-direction. For two arbitrary components, their projections on the same plane lead to one of two possible spatial relationships: either they overlap or they do not. It should be noticed that if the overlap is not a 22 face, it is regarded as no overlap case. If their projections overlap, we will compute the region of intersection. For instance, Figure 3 illustrates four different cases, in which the original surfaces are $A$ and $B$, and $\alpha$ and $\beta$ are their projections on the same plane respectively. Then their overlaps are computed by region intersection. Based on the overlaps of their projections, the effective upper and lower boundaries can be determined. This process is quantified by equation 2 . It should be noticed that $A^{\prime}$ and $B^{\prime}$ in the equation are part of areas in surface $A$ and $B$ respectively, acting as effective upper and lower boundaries. Projections of $A^{\prime}$ and $B^{\prime}$ are equal to $\alpha \cap \beta$. $A^{\prime}$ and $B^{\prime}$ can be calculated by projecting their overlapping area $\alpha \cap \beta$ back to $A$ and $B$ along the Z-direction.

$$
\left\{\begin{aligned}
\alpha=\beta & U=A, L=B \\
\alpha \subset \beta & U=A, L=B^{\prime} \\
\beta \subset \alpha & U=A^{\prime}, L=B \\
\alpha \cap \beta \neq \emptyset & U=A^{\prime}, U=B^{\prime}
\end{aligned}\right.
$$

where

$$
\begin{aligned}
& \alpha=\text { the projection of component (surface) A } \\
& \beta=\text { the projection of component (surface) B } \\
& U=\text { the upper boundary } \\
& L=\text { the lower boundary } \\
& A^{\prime}=\text { the area in surface } A, \text { whose projection is } \alpha \cap \beta
\end{aligned}
$$$$
B^{\prime}=\text { the area in surface } B \text {, whose projection is } \alpha \cap \beta
$$$$
36
$$

In the case where the projections have no overlap, it means that the selected pair of surfaces does not form a top bounded space together. Thus, the overlap will be sought between $A$ and a surface at a lower height if any, while $B$ will be processed similarly to $A$ at a later stage. If there is no more surface component at a lower height, the default lower surface is the Ground.

\section{Algorithm 1: Upper and lower boundary determination \\ Input: A list of sorted surfaces based on elevation $S$, in which each component (surface) is $S_{i}$. The default ground $G$}

Output: A list of boundary combinations $B$

$1 U=S_{0}$ \# Upper boundary $U$

$2 L=G$ \# Lower boundary $L$

3 temp $=0$

4 for each $S_{i}$ in $S$ do

$5 \quad$ temp $=$ temp +1

RegionIntersection(projection 1 , projection 2 ) if $R$ Int then

\# if the intersection is not null

$S_{i}^{\prime}=$ ProjectOnSurface $\left(R I n t, S_{i}\right)$

$S_{j}^{\prime}=$ ProjectOnSurface $\left(R I n t, S_{j}\right)$

RDiff $1=$

RegionDifference(projection 1 , projecttion 2 )

RDiff $2=$

RegionDifference(projection 2 , projection 1 )

if $R D$ iff 1 \&\& RDiff 2 then

$$
\begin{aligned}
& \# S_{i} \cap S_{j} \neq \emptyset \\
& U=S_{i}^{\prime}, L=S_{j}^{\prime} \\
& \text { Add the pair }[i, j,\{U, L\}] \text { in } B[i]
\end{aligned}
$$

\section{else if}

(RDiff $1 \& \& ! R D i f f 2) \|(! R D i f f 1 \& \& R D$ iff 2$)$

then

if ! RDiff $1 \& \&$ RDiff 2 then

$\# S_{i} \subset S_{j}$

$U=S_{i}, L=S_{j}$

Add the pair $[i, j,\{U, L\}]$ in $B[i]$

break

else

$\# S_{j} \subset S_{i}$

$U=S_{i}^{\prime}, L=S_{j}$ Add the pair $[i, j,\{U, L\}]$ in $B[i]$

else

$$
\# S_{i}=S_{j}
$$

$U=S_{i}, L=S_{j}$

Add the pair $[i, j,\{U, L\}]$ in $B[i]$

break 
Based on the relationships of two surfaces, the upper and lower boundaries of all of the spaces can be determined by Algorithm 1, in which, $S_{i}^{\prime}$ and $S_{j}^{\prime}$ are the areas in surface $S_{i}$ and $S_{j}$, which projections are equal to $S_{i} \cap S_{j}$. The $S_{i}^{\prime}$ can be calculated by projecting the overlap of their projection onto $S_{i}$ along the Zdirection, so does $S_{j}^{\prime}$. The output of this algorithm is a list of boundary combinations $B$. Each element $B[i]$ in $B$ is a threetuple, e.g., $B[i]=<i, j,\{U, L\}>$, in which the $i$ is the $I d$ of the upper boundary, and the $j$ is the $I d$ of the lower boundary. For the ground, we use the 'Ground' directly as a $I d .\{U, L\}$ is the geometry pair of the surfaces $i$ and $j$.

The functions used in the algorithm are the following:

ProjectAlong(obj): project object obj on the XY plane along the Z-direction.

RegionIntersection(obj1,obj2): calculate the intersection between $o b j 1$ and $o b j 2$, which are two coplanar closed surfaces.

RegionDifference(obj1,obj2): calculate the difference between $o b j 1$ and $o b j 2$, which are two coplanar closed surfaces.

ProjectOnSurface(obj1,obj2): calculate the area in obj2 by projecting $o b j 1$ on the $o b j 2$ along the Z-direction, in which $o b j 1$ and $o b j 2$ are two closed surfaces.

\subsection{Extraction \& Trimming of Spaces}

This step creates the 3D volume of the space based on the combination of the identified upper and lower boundaries. Figure 4 illustrates the results on the surfaces described in Figure 3. Because our approach relies entirely on the Z-direction as projection direction, the upper and lower boundaries can be directly matched to obtain the missing lateral surfaces that allow forming closed volumes of the spaces.

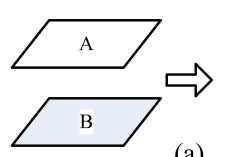

(a)
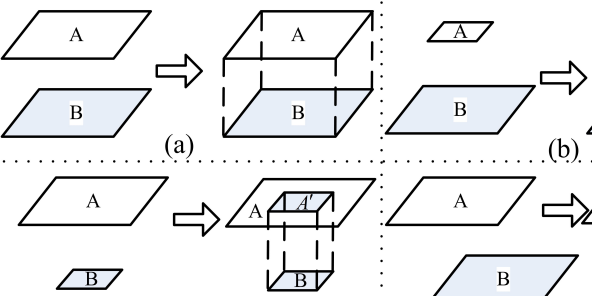

(c)

(d)

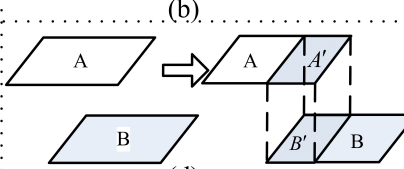

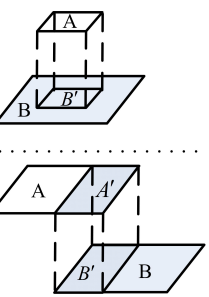

Figure 4. Space creation between two arbitrary surfaces.

A consequence to this method is that the resulting volumes may also overlap. Such case is illustrated in Figure 5, where there are three surfaces $A$ (roof), $B$ (floor slab), and $C$ (ground/floor) represented. If $\alpha, \beta$, and $\gamma$ are their projections respectively, their relationships are $\alpha \cap \beta \neq \emptyset, \alpha \subset \gamma$, and $\beta \subset \gamma$. Therefore, there are three top-bounded spaces that will be generated by our approach: one between $A$ and $B$ (see Figure 5(b)), between $A$ and $C$ (see Figure 5(c)), and one between $B$ and $C$ (see Figure 5(d)). However, the space bounded by $A$ (upper boundary) and $C$ (lower boundary) should exclude the parts resulting from the spaces in Figure 5(b) and (c). Therefore, the trimming process allows to obtain the final space described in Figure 5(e). This way, the three final top-bounded spaces can be seen in Figure $5(f)$.

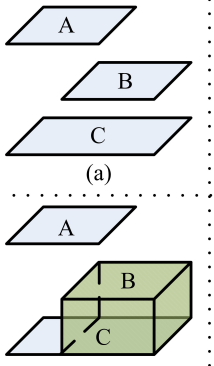

(d)

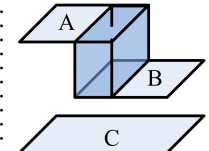

(b)

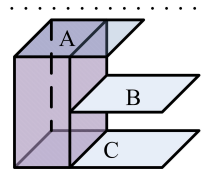

(e)

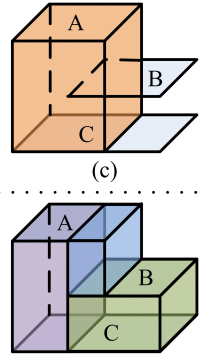

Figure 5. Example of trimming spaces.

The Algorithm 2 shows the details of the whole process of creating and trimming the spaces. The function CreateBooleanDifference (obj1, obj2, $t$ ) that is used computes the Boolean difference between $o b j 1$ and $o b j 2$, taking the tolerance $(\mathrm{t})$ into consideration. obj1 and $o b j 2$ are assumed to be two closed volumes.

Algorithm 2: The process of creating and trimming topbounded spaces based on their upper and lower boundary.

Input: A list of sorted surfaces based on elevation $S$, Boundary combinations set $B$, Sorted surfaces based on elevation $S_{n}$

Output: Top-bounded spaces $R$

1 Space $=[]$

2 for each $b_{i}$ in $B$ do

for each temp in $b_{i}$ do

$r_{i}=$ CreateSpace $($ temp $\{U, L\})$

Add the pair $\left[B[0], B[1], r_{i}\right]$ to Space

for each $i \in[0$, Size of $S]$ do

temp $=[]$

for each $k \in[0$, Size of Space $]$ do

if Result $[k][0]==i$ then

L Add Space[k][2] in temp

if Size of temp $==1$ then

Add temp [0] in $R$

else

flag $=$ Size of temp -1

for $e a c h j \in[0$, flag $]$ do

Add temp $[j]$ in $R$

$o b j 1=$ temp $[$ flag $]$

$o b j 2=\operatorname{temp}[j]$

middleSpace $=$

CreateBooleanDifference $(o b j 1, o b j 2, t)$

Add middleSpace in $R$

return $R$

All figure drew in above gave the impression that proposed approach is only for the situation where components (surfaces) are horizontal. In fact, this method does not have any problems when dealing with inclined surfaces, except the vertically down components, as the projection of vertical components on the Z-direction is a line rather than a surface. Figure 6 illustrates the inclined cases. There are five surfaces selected to extract the top-bounded spaces, Roof 1, Balcony 1, Balcony 2, Roof 2, and Ground, in which the Roofs and Ground are inclined surfaces, see $(a)$. Then the projections can be calculated based proposed approach, thus six top-bounded spaces $(A$ to $Z$ ) are extracted, see $(b)$ and $(c)$. All the spaces generated can be seen in $(d)$. 


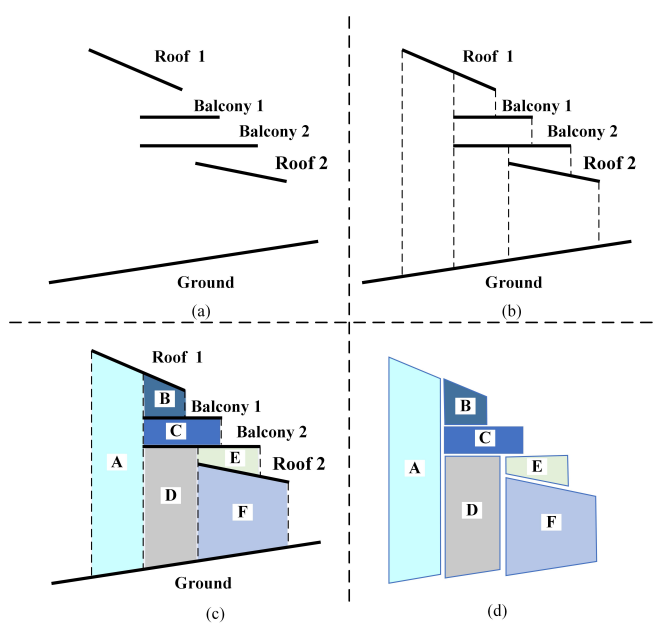

Figure 6. Side view of top-bounded spaces extraction with inclined components (surfaces).

\section{IMPLEMENTATIONS AND EXPERIMENTS WITH CITYGML}

Top-bounded spaces can be extracted based on our proposed approach. In the CityGML data model, several classes available in that standard makes it interesting to work with for the purpose of detecting semi-bounded spaces. Building elements like balconies, chimneys, dormers or outer stairs are represented by the BuildingInstallation class. The shell based representation provided by the standard gives direct access to interesting components such as RoofSurface, GroundSurface, OuterCeilingSurface and OuterFloorSurface, as illustrated in Figure 7. Those latter elements are allowed to be encapsulated or referenced by the boundedBy property of BuildingInstallation, along with WallSurface and ClosureSurface elements.
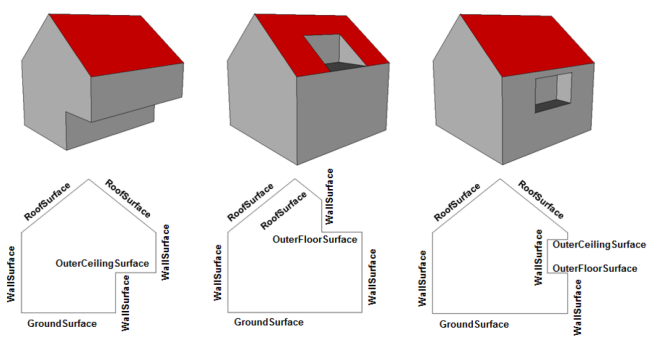

Figure 7. Buildings components in CityGML shell model. (Gröger et al., 2008)

Other elements of interest, such as FloorSurface, CeilingSurface, InteriorWallSurface, and ClosureSurface are more relevant to the indoor case. For this reason, they are allowed to be encapsulated or referenced by the boundedBy property of the Room class. Thus, the classes BuildingInstallation (top-bounded) and Room (indoor) are the main elements to analyze in the model, to find the semi-bounded spaces.

It is important to mention that the most of the cases where top bounded spaces can be detected will likely appear in LoD3 (and above) models, although some LoD2 models may also present interesting cases as well (see Figure 7). However, due to the lack of availability of LoD3 or LoD4 data, we represented a synthetic LoD4 model for the sake of illustration of the approach (see Figure 8). We then identified the buildings components of

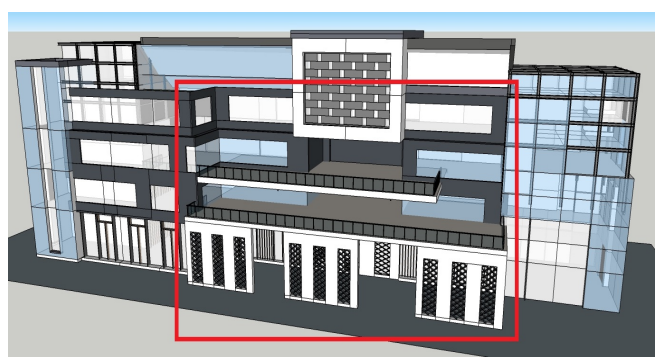

Figure 8. A 3D building model.

interest such as roofs (RoofSurface), floor slabs (OuterCeilingSurface), floors (OuterFloorSurface), and ground (GroundSurface). These components can be obtained from a CityGML model parser. Then, they can be sorted out on the basis of their height as discussed in the method.

In the chosen scene, there are three tops (two OuterFloorSurface, and one OuterCeilingSurface), see Figure 9. Theoretically, three top-bounded spaces will be brought in by these tops.

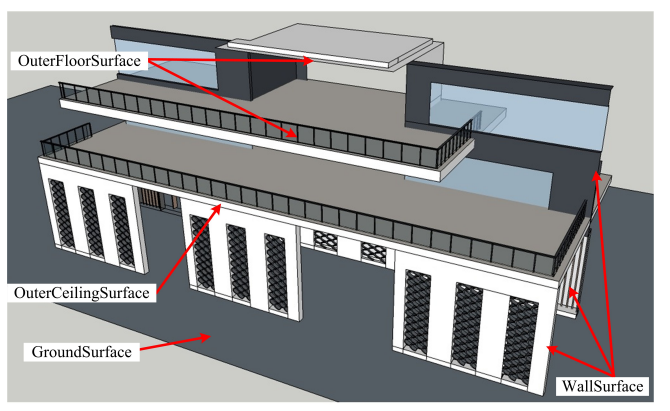

Figure 9. Simplified 3D model with top-bounded structures only.

CityGML represents 3D geometry according to the well-known Boundary Representation (B-Rep) to avoid redundancy. Thus, the components of our 3D building can be further simplified as Surface, see Figure 10.

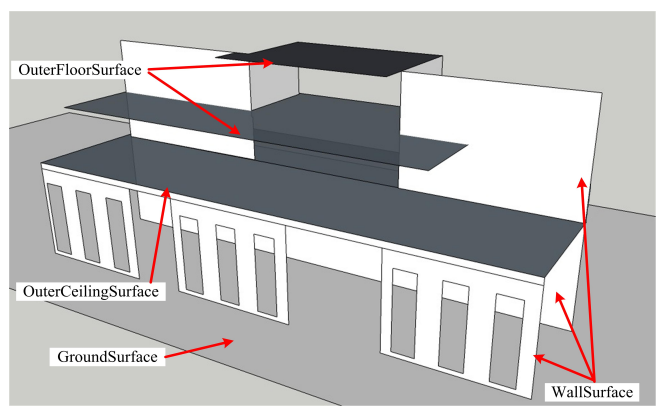

Figure 10. Simplified 3D CityGML model with top-bounded structures only.

The projections of the components $(A, B, C$, and Ground), as represented in Figure 11, are $\alpha, \beta, \gamma$, and $\theta$. The location of the components can be seen in Figure 11. Then, the relationships are $\alpha \subset \beta \subset \gamma \subset \theta$. Therefore, only three top-bounded spaces can be created based on the upper\&lower combinations, i.e., $\left(U_{1}, L_{1}\right)=\{A, B\},\left(U_{2}, L_{2}\right)=\{B, C\}$, $\left(U_{3}, L_{3}\right)=\{C$, Ground $\}$. 


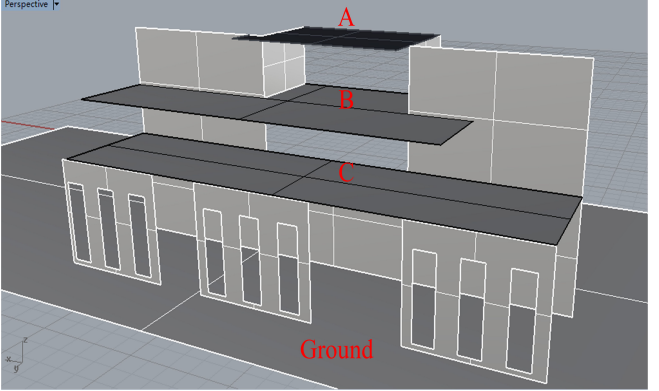

Figure 11. Identified experimental components for top-bounded space creation.

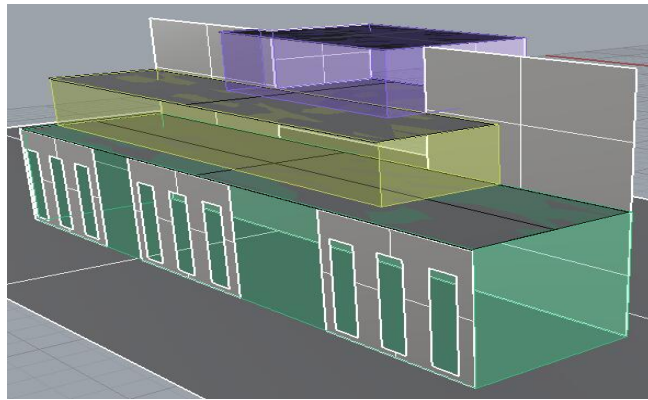

Figure 12. Top-bounded spaces creation in Rhino.

In this paper, we conducted the experiments on a 3D model by using the Rhinoceros + Grasshopper software, and the whole data process is developed in Python script. Functions, including ProjectAlong, RegionIntersection, RegionDifference, CreateSpace, and CreateBooleanDifference comes from RhinoCommon SDK, see Figure 12. No trimming process is needed as these three topbounded spaces have no overlaps. Therefore, the final results are three top-bounded spaces, see Figure 13.

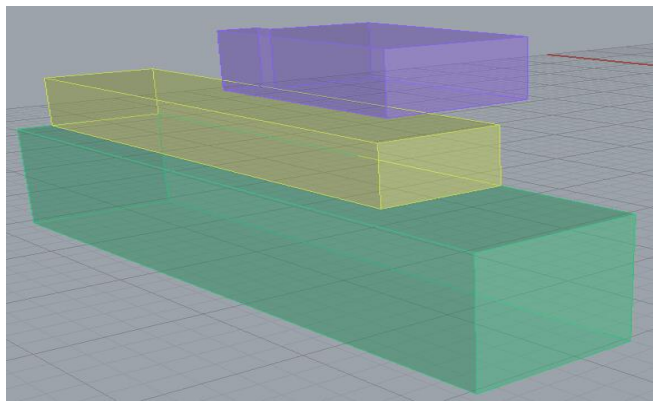

Figure 13. Created top-bounded spaces by proposed approach

With the same approach, the top-bounded spaces of the built structures like the gas station (Figure 1(c)), and bus stop (Figure 1(b)), can be generated. Specifically, their roofs (covers/shelters) are used as the upper boundaries, and the GroundSurface as lower boundary. Besides BuildingInstallation, another interesting case of top-bounded environment is a tunnel. It can never be enclosed completely like a room because of its entrances. Thus in CityGML, it is subdivided into one Tunnel (the actual underpass) and two TunnelParts (both entrances). Tunnel and tunnel parts are bounded by GroundSurface, WallSurface, RoofSurface. ClosureSurface objects are used to virtually seal the tunnel entrances. For safety reasons each of the two entrances has railings which are modelled as TunnelInstallation.

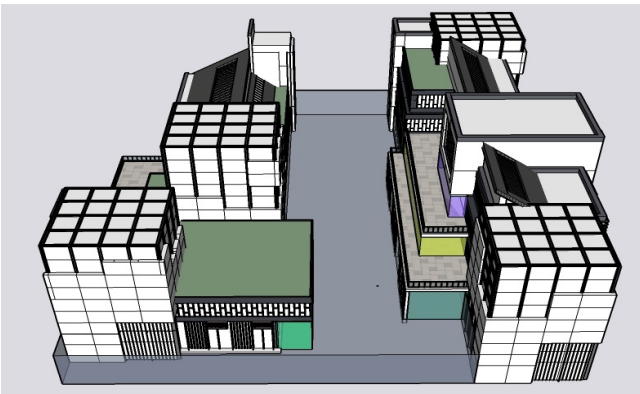

Figure 14. A street formed by two buildings.

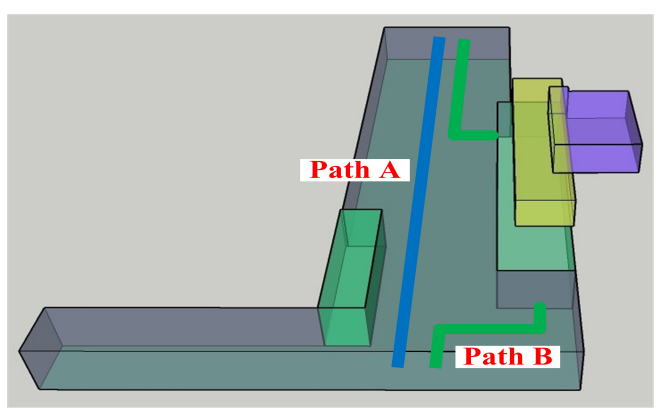

Figure 15. Top-bounded spaces and the corresponding navigation paths.

After demonstrating how to create the top-bounded spaces based proposed approach, the Figure 14 and 15 show how this type of space is used in pedestrian navigation. In Figure 14, there is a street formed by two buildings, in which the building on the right side is the one shown in Figure 8. The outdoor space is coloured as grey. Two paths are shown in Figure 15. The Path $A$ is the navigation path in current navigation (systems) without considering the top-bounded spaces, but the Path $B$ takes these top-bounded spaces into account. The latter path could be a better choice for pedestrian when they need shelter to escape rain or strong sun.

\section{CONCLUSION AND FUTURE WORK}

In this paper, we concentrated on the identification of semibounded spaced and presented an automatic method for topbounded spaces creation from 3D models. Specifically, the method is based on sorting if navigation surfaces and checking the projections of their intersections. The results of the implementation and experiments indicate that the generation of topbounded spaces (formed by built structures) is feasible on existing 3D standards such as CityGML or IFC.

The focus of this approach was on the geometric aspect to determine top-bounded spaces. However, a more detailed semantic information such as the function of those spaces (shelters, sun protections, decorations) can be further investigated and used for the space identification. For instance, pedestrians may need the shelters to escape from the rain, while some tops which are more decorative-oriented (with holes, irregular shapes, etc.) cannot meet such needs. Therefore, the top-bounded space from decoration tops should be neglected. Furthermore, we mainly dealt with spaces formed by flat built structures, i.e., all the surfaces are flat, rather than curved. But in the real world, the top can be formed 
by curved built structures (e.g., tunnel with arc cross-section). Our approach is still able to handle such cases, but geometric and implementation adaptations might be still needed.

The proposed geometry based top-bounded space creation is not yet taking into consideration the dimensions of the pedestrians. Considering length of the pedestrians may lead to the discarding of some top-bounded spaces which are too small or too high to provide protection, or even somewhere pedestrian cannot visit, for instance, the space $E$ in Figure 6. But their impact should be evaluated beforehand in future research. Finally, the usage of the terrain spaces and semantics is not considered yet. For instance, if water (or road) on one side of the building, the top-bounded spaces formed by the building (upper boundary) and water (lower boundary) cannot be used for pedestrian navigation.

\section{ACKNOWLEDGEMENTS}

The financial support for this work comes from the program of China Scholarships Council (No. 201606410054).

\section{REFERENCES}

Building SMART International, 2013. Industry Foundation Classes (IFC), IFC4.

Diakité, A. A. and Zlatanova, S., 2018. Spatial subdivision of complex indoor environments for $3 \mathrm{~d}$ indoor navigation. International Journal of Geographical Information Science 32(2), pp. 213-235.

Du, X., Bokel, R. and van den Dobbelsteen, A., 2014. Building microclimate and summer thermal comfort in free-running buildings with diverse spaces: A chinese vernacular house case. Building and Environment 82, pp. 215-227.

Gröger, G., Kolbe, T. H., Czerwinski, A. and Nagel, C., 2008. Opengis city geography markup language (citygml) encoding standard, version 1.0. 0.

He, J. and Hoyano, A., 2010. Measurement and evaluation of the summer microclimate in the semi-enclosed space under a membrane structure. Building and Environment 45(1), pp. 230-242.

Kim, J., Kim, T. and Leigh, S.-B., 2011. Double window system with ventilation slits to prevent window surface condensation in residential buildings. Energy and Buildings 43(11), pp. 31203130.

Kim, K., Park, S. and Kim, B. S., 2008. Survey and numerical effect analyses of the market structure and arcade form on the indoor environment of enclosed-arcade markets during summer. Solar Energy 82(10), pp. 940-955.

Koide, S. and Kato, M., 2005. 3-d human navigation system considering various transition preferences. In: Systems, Man and Cybernetics, 2005 IEEE International Conference on, Vol. 1, IEEE, pp. 859-864.

Kray, C., Fritze, H., Fechner, T., Schwering, A., Li, R. and Anacta, V. J., 2013. Transitional spaces: between indoor and outdoor spaces. In: International Conference on Spatial Information Theory, Springer, pp. 14-32.

Krūminaitè, M. and Zlatanova, S., 2014. Indoor space subdivision for indoor navigation. In: Proceedings of the Sixth ACM SIGSPATIAL International Workshop on Indoor Spatial Awareness, ACM, pp. 25-31.
Lee, J., Li, K.-J., Zlatanova, S., Kolbe, T., Nagel, C. and Becker, T., 2014. Ogc indoorgml. Open Geospatial Consortium standard.

Lin, T.-P., 2009. Thermal perception, adaptation and attendance in a public square in hot and humid regions. Building and environment 44(10), pp. 2017-2026.

Lin, T. P., Matzarakis, A. and Huang, J. J., 2006. Thermal comfort and passive design strategy of bus shelters.

May, A. J., Ross, T., Bayer, S. H. and Tarkiainen, M. J., 2003. Pedestrian navigation aids: information requirements and design implications. Personal and Ubiquitous Computing 7(6), pp. 331338 .

Melson, G. F., 1977. Sex differences in use of indoor space by preschool children. Perceptual and Motor Skills 44(1), pp. 207213.

Nagel, C., Becker, T., Kaden, R., Li, K., Lee, J. and Kolbe, T. H., 2010. Requirements and space-event modeling for indoor navigation. Open Geospatial Consortium.

Nasir, N. H. M., Salim, F. and Yaman, M., 2014. The potential of outdoor space utilization for learning interaction. UMRAN2014: Fostering Ecosphere in Built Environment, International Islamic University Malaysia pp. 1-17.

Neufert, E., Neufert, P. and Kister, J., 2012. Architects' data. John Wiley \& Sons.

Pagliarini, G. and Rainieri, S., 2011a. Dynamic thermal simulation of a glass-covered semi-outdoor space with roof evaporative cooling. Energy \& Buildings 43(23), pp. 592-598.

Pagliarini, G. and Rainieri, S., 2011b. Thermal environment characterisation of a glass-covered semi-outdoor space subjected to natural climate mitigation. Energy and Buildings 43(7), pp. 1609-1617.

Ruey-LungHwang and Tzu-PingLin, 2007. Thermal comfort requirements for occupants of semi-outdoor and outdoor environments in hot-humid regions. Architectural Science Review 50(4), pp. 357-364.

Spagnolo, J. and Dear, R. D., 2003. A field study of thermal comfort in outdoor and semi-outdoor environments in subtropical sydney australia. Building \& Environment 38(5), pp. 721-738.

Taj, H. V. and Blocken, B., 2009. Computational analysis of natural ventilation in a large semi-enclosed stadium. Firenze University Press.

Turrin and Timmeren, V., 2009. Case study 'the vela roof unipol', bologna use of on-site climate and energy resources. In: Energy \& Sustainability.

Turrin, M., Buelow, P. V., Kilian, A. and Stouffs, R., 2012. Performative skins for passive climatic comfort : A parametric design process. Automation in Construction 22(4), pp. 36-50.

Turrin, M., Buelow, P. V., Stouffs, R. and Kilian, A., 2010. Performance-oriented design of large passive solar roofs.

Winter, S., 2012. Indoor spatial information. International Journal of 3-D Information Modeling (IJ3DIM) 1(1), pp. 25-42.

Worboys, M., 2011. Modeling indoor space. In: Proceedings of the 3rd ACM SIGSPATIAL International Workshop on Indoor Spatial Awareness, ACM, pp. 1-6.

Zlatanova, S., Liu, L., Sithole, G., Zhao, J. and Mortari, F., 2014. Space subdivision for indoor applications. GISt Report No. 66. 